\title{
Perfil das ceratites fúngicas no Hospital São Geraldo Belo Horizonte - MG
}

\author{
Spectrum of fungal keratitis at the São Geraldo Hospital-Belo Horizonte -MG
}

\author{
Cristina Moreira Salera ${ }^{1}$ \\ Marco Antônio Guarino Tanure ${ }^{2}$ \\ Wellington Tadeu Monteneg'ro Lima ${ }^{3}$ \\ Christian Marcellus Campos ${ }^{1}$ \\ Fernando Cançado Trindade ${ }^{4}$ \\ Júlia dos Anjos Moreira ${ }^{5}$
}

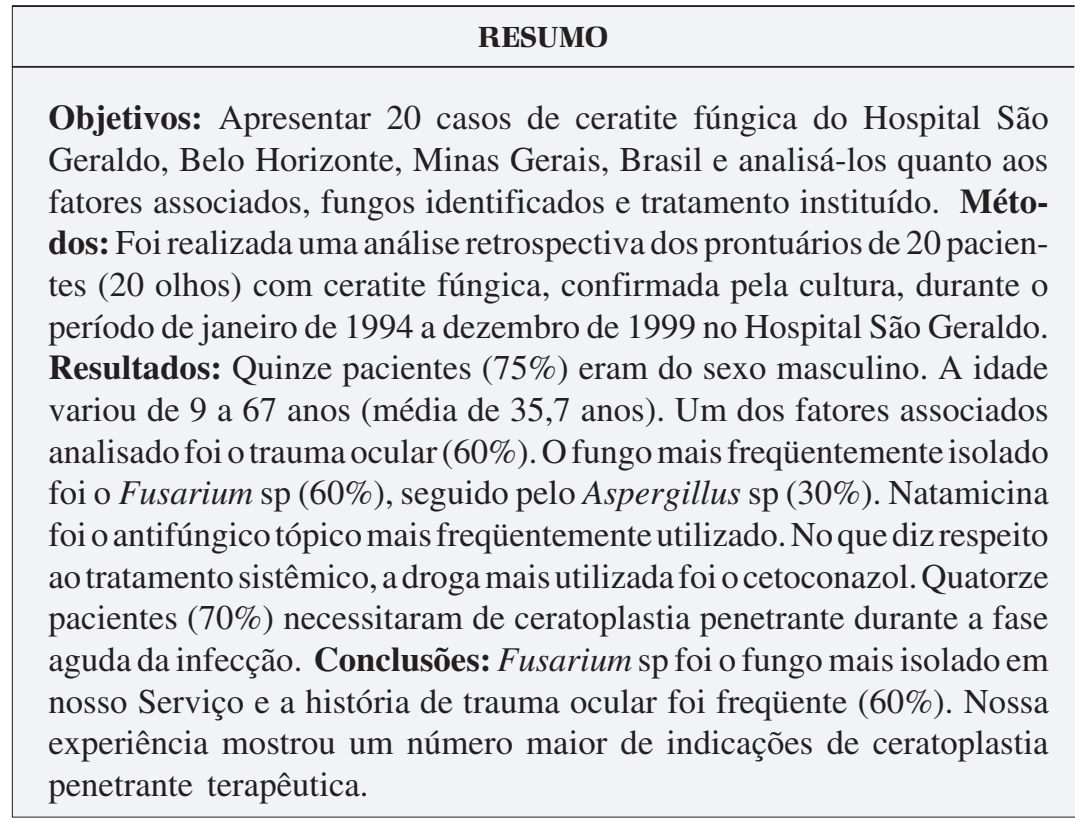

Descritores: Ceratite/quimioterapia; Infecções oculares fúngicas/quimioterapia; Córnea/ lesões

\section{INTRODUÇÃ̃O}

O primeiro caso de ceratite fúngica foi descrito por Leber em $1879^{(1)}$. Levantamentos recentes mostram um aumento significativo dos casos de ceratomicose nas últimas quatro décadas. Este fato pode ser atribuído ao desenvolvimento e ao uso indiscriminado de antibióticos de amplo espectro e de corticóides, uso de lentes de contato, aumento do número de cirurgias corneanas e aos avanços nas técnicas de diagnóstico ${ }^{(2-9)}$.

A epidemiologia da ceratomicose depende de fatores climáticos ${ }^{(2,8-12)}$. Em climas temperados (Europa Central, Inglaterra, norte dos EUA), Candida sp é o fungo isolado com mais frequiência, seguido pelo Aspergillus $\mathrm{sp}^{(2,5-6,8-9,13-14)}$. Já em regiões tropicais (Sul da Flórida, América do Sul, Japão, Nigéria, África do Sul), o fungo mais freqüentemente encontrado é o Fusarium $\mathrm{sp}^{(2-3,5-5,8-9,9,11,13-14)}$. $\mathrm{O}$ fungo mais isolado em todo mundo é o Aspergillus $\mathrm{sp}^{(2-3,6,14)}$.

Os fungos filamentosos estão associados ao trauma com vegetal, enquanto os fungos leveduriformes são tipicamente encontrados em pacientes com doença ocular ou sistêmica prévia ${ }^{(3-4,7-9,11-12,14)}$.

Com relação à biota conjuntival, é relatado que os fungos são isolados do saco conjuntival de olhos saudáveis em uma incidência que varia de 2,9\% a $72 \%^{(7-9,12,15-19)}$. Os fungos mais freqüentemente isolados nesses olhos são: 
Aspergillus sp, Candida sp, Penicillium sp, Cladosporium $\mathrm{sp}$ e Alternaria $\mathrm{sp}^{(6,20)}$. Uma incidência de $17 \%$ a $37 \%$ é obtida em olhos doentes ${ }^{(6)}$. A presença de fungos patogênicos encontrados na conjuntiva normal de cortadores de cana de açúcar em regiões distintas do interior do Brasil correspondeu a uma variação de $38,5 \%$ a $72 \%{ }^{(9,12,17)}$ O trabalho de Willianson de 1965 sugere que o fungo seja mais um contaminante transitório do que um comensal, uma vez que o fungo não permanece ou cresce no saco conjuntival de olhos normais ${ }^{(16,21)}$.

O presente trabalho tem como objetivos caracterizar os fungos identificados e os fatores associados, bem como avaliar os aspectos clínicos, o tratamento instituído, a evolução e as complicações dos casos de ceratite micótica diagnosticados em serviço oftalmológico de referência, com localização no município de Belo Horizonte (MG), região sudeste do Brasil.

\section{MÉTODOS}

Foram avaliados retrospectivamente 20 prontuários de pacientes atendidos e acompanhados no Serviço de Córnea e Doenças Externas Oculares do Hospital São Geraldo da Universidade Federal de Minas Gerais (UFMG). Todos eles apresentaram diagnóstico de ceratite fúngica com identificação do fungo em meio de cultura, segundo as normas técnicas do Laboratório de Microbiologia da Faculdade de Medicina da $\mathrm{UFMG}^{(22)}$ durante o período de janeiro de 1994 a dezembro de 1999. Ressaltamos que não houve identificação da espécie dos fungos isolados, houve identificação apenas do gênero e que estes são todos os casos tratados no período. Em todos os casos, foi realizado raspado corneano e o material obtido corado por Gram, Giemsa e semeado nos meios de cultura ágar - sangue, ágar - chocolate, ágar - Sabouraud e tioglicolato.

Os critérios para indicação de ceratoplastia penetrante foram a presença de perfuração corneana e a ausência de resposta ao tratamento clínico. A injeção intravítrea de antimicótico foi indicada na presença de inflamação intra-ocular e o recobrimento conjuntival foi indicado nos casos em que houve progressão do quadro apesar das medicações ou mesmo após transplante terapêutico.

Os dados avaliados nos referidos prontuários foram:

- sexo;

- idade;

- procedência;

- profissão;

- agente etiológico;

- período entre o início das queixas e a entrada no Serviço de Córnea;

- fatores associados: traumatismo ocular e uso de medicação tópica prévia;

- sinais clínicos;

- tratamento inicial instituído;

- necessidade de substituição do agente antifúngico;
- uso de antifúngico sistêmico;

- indicação terapêutica de transplante de córnea;

- recidiva da infecção fúngica no enxerto corneano;

- outros procedimentos cirúrgicos.

\section{RESULTADOS}

Dos 20 casos avaliados, 15 (75\%) eram pacientes do sexo masculino e 5 (25\%), do sexo feminino. A idade desses pacientes variou de 9 a 67 anos (média de 35,7 anos). Analisando-se a procedência, verificou-se que $15(75 \%)$ pacientes eram do interior do estado de Minas Gerais e cinco (25\%) eram de outros estados, sendo quatro desses da região norte do Brasil. No que diz respeito à profissão, $12(60 \%)$ pacientes eram lavradores, três $(15 \%)$ eram donas de casa, três $(15 \%)$ eram estudantes, um (5\%) era aposentado, e um $(5 \%)$ era pedreiro.

Com relação à etiologia das ceratites, foram $12(60 \%)$ casos de Fusarium sp, seis (30\%) casos de Aspergillus sp, um (5\%) caso de Acremonium sp e um (5\%) caso de Drechesclera sp. Nenhum fungo leveduriforme foi identificado no período.

O período entre o início dos sinais e sintomas e a chegada ao Serviço de Córnea variou de 12 a 90 dias (média de 26,5 dias), excetuando-se um paciente que apresentou perfuração corneana imediata durante o trauma com vegetal (caso 10) e outros três pacientes que não souberam informar quando as queixas se iniciaram (casos 5, 19 e 20).

Quanto aos fatores associados pesquisados, o traumatismo ocular esteve presente em $12(60 \%)$ pacientes. Oito (40\%) não apresentaram relato de traumatismo ocular.

Dos casos estudados, 17 (85\%) tinham antecedentes de uso de medicação tópica antes de sua admissão no Serviço de Córnea. Sendo que $12(70,6 \%)$ pacientes fizeram uso de antibacteriano tópico e um $(5,9 \%)$ paciente fez uso de antibacteriano associado a corticóide tópico. Quatro $(23,5 \%)$ pacientes não souberam informar o nome dos colírios utilizados. Três (15\%) não informaram quanto à existência de tratamento prévio.

Os achados clínicos mais freqüentes foram a presença de congestão pericerática (100\%), defeito epitelial (100\%) e infiltrado estromal (100\%). A reação de câmara anterior foi encontrada em $10(50 \%)$ pacientes. Características da lesão corneana como textura seca e bordas plumosas foram detectadas em nove $(45 \%)$ pacientes e as bordas elevadas foram observadas em cinco (25\%) casos. Hipópio, lesões satélites, dobras na Descemet e inflamação intra-ocular foram encontradas em oito (40\%), dois (10\%), um (5\%) e um (5\%) casos respectivamente.

Os sinais clínicos mais freqüentes estão descritos na tabela 1.

Uma vez admitidos no Serviço de Córnea, o tratamento inicial foi com natamicina tópica em 17 (85\%) pacientes, miconazol tópico em um (5\%) paciente (caso 2) e em dois (10\%) casos com injeção intravítrea de anfotericina $\mathrm{B}$ devido à presença de inflamação intra-ocular (casos 10 e 14). O tempo do uso da natamicina tópica variou de 20 a 180 dias, com média de 75,3 dias. 


\begin{tabular}{|lcc|}
\hline \multicolumn{3}{|c|}{ Tabela 1. Sinais clínicos mais freqüentes } \\
Sinais Clínicos & Número de Casos & $\%$ \\
Congestão pericerática & 20 & 100 \\
Defeito epitelial & 20 & 100 \\
Infiltrado estromal & 20 & 100 \\
Reação de câmara anterior & 10 & 50 \\
Bordas plumosas & 9 & 45 \\
Textura seca & 9 & 45 \\
Hipópio & 8 & 40 \\
Bordas elevadas & 5 & 25 \\
Lesões satélites & 2 & 10 \\
Dobras na Descemet & 1 & 5 \\
Inflamação intra-ocular & 1 & 5 \\
\hline
\end{tabular}

Dos pacientes que iniciaram o tratamento com natamicina, em $13(76,5 \%)$ não foi necessária a opção por outra medicação, em quatro $(23,5 \%)$ pacientes o tratamento tópico foi mudado para anfotericina B. O paciente que iniciou o seu tratamento com miconazol tópico (caso 12) também teve sua prescrição alterada para anfotericina B. Nestes cinco pacientes (casos 6, $9,11,12,20)$ em que foi indicada a troca da medicação devido à progressão do quadro infeccioso, os fungos identificados foram os seguintes: Fusarium sp em três $(60 \%)$ pacientes (casos 6, 9, 20), Aspergillus sp em um (20\%) paciente (caso 12) e Drechesclera sp em um (20\%) paciente (caso 11). Com relação à evolução destes casos, é interessante ressaltar que dos cinco pacientes em que a alteração da medicação foi necessária, quatro $(80 \%)$ evoluíram para ceratoplastia penetrante terapêutica (casos $6,9,12,20)$ e todos eles tiveram recidiva da infecção fúngica no enxerto.

A medicação sistêmica foi utilizada em $16(80 \%)$ pacientes, sendo $12(75 \%)$ deles tratados com cetaconazol, e quatro (25\%), com itraconazol. Em quatro (20\%) não foi realizado tratamento sistêmico. Dos casos que usaram itraconazol, em um deles fez-se necessário o uso de anfotericina B sistêmica devido à gravidade do quadro de endoftalmite (caso 10).

Houve necessidade de realização de ceratoplastia penetrante terapêutica em 14 (70\%) casos, enquanto seis (30\%) pacientes receberam apenas tratamento clínico. Dos casos submetidos ao transplante corneano, em $13(92,8 \%)$ a indicação foi devido à perfuração corneana e um $(7,2 \%)$, por ausência de resposta ao tratamento (caso 3). Houve recidiva da infecção na córnea transplantada em sete $(50 \%)$ casos. Dos casos recidivados, três $(42,9 \%)$ melhoraram com tratamento clínico (casos 9,15 e 20) e em três $(42,9 \%)$ casos houve indicação de um segundo transplante terapêutico (casos 6, 12 e 13), havendo também um caso $(14,2 \%)$ com indicação de recobrimento conjuntival parcial (caso 16). Dos pacientes que foram submetidos ao segundo transplante terapêutico, em um caso foi necessária a realização de um terceiro transplante corneano seguido de recobrimento conjuntival total (caso 6).

A tabela 2 mostra os dados clínicos e a evolução dos pacientes.

\section{DISCUSS $\tilde{A} O$}

No presente estudo, observou-se um maior número de pacientes do sexo masculino acometidos por ceratites fúngicas. Este fato pode ser atribuído à maior exposição do homem aos fatores predisponentes ${ }^{(6-7,10-11,18,23-24)}$. A idade média foi de 35,7 anos, caracterizando o acometimento na faixa etária de maior atividade $^{(6,7,11,23)}$. Verificou-se que a procedência de $100 \%$ dos casos era do meio rural e $60 \%$ eram lavradores, demonstrando a importância na etiologia da úlcera fúngica do contato com o meio agrário e a suscetibilidade ao trauma ocular com vegetais ${ }^{(3,5,7,9,11-13,17-18,23,25-26)}$. Este último fator foi verificado em $60 \%$ dos casos. A literatura mundial associa o trauma a ceratites fúngicas em $33 \%$ a $100 \%$ dos pacientes ${ }^{(2,5-6,9,11,13,25,27)}$.

Salienta-se que nos casos analisados houve predominância do trauma gerando doenças caracterizadas como agudas e, conseqüentemente, sem fatores crônicos predisponentes à instalação de outros fungos como, por exemplo, Candida sp.

Outro fator associado observado foi o uso prévio de antibacteriano tópico $(70,6 \%)$ ou antibacteriano associado a corticóide tópico $(5,9 \%)$. A descrição deste achado na literatura variou de $12,8 \%$ a $87 \%{ }^{(6,8,11,24,28)}$. Esta grande porcentagem de pacientes com antecedentes de uso de medicação tópica se deve ao fato de que, por se tratar de um serviço de referência, nos são encaminhados casos mais graves e aqueles sem resposta positiva ao tratamento inicialmente instituído.

O período entre o início dos sinais e sintomas e a entrada no Serviço de Córnea variou em média 26,5 dias o que pode ser justificado pela procedência rural dos pacientes, pelas dificuldades no diagnóstico e nos tratamentos empíricos instituídos bem como o encaminhamento ao serviço especializado já em estágio avançado de comprometimento ocular.

Os sinais clínicos analisados neste estudo não diferem de outros relatos da literatura ${ }^{(2,6,9,21,23,25)}$, excetuando-se a maior ocorrência de hipópio, o que se justifica pela demora no acesso ao serviço médico especializado devido à procedência dos pacientes. É interessante ressaltar que o trabalho de Srinivasan et al. (1991) ${ }^{(23)}$ correlacionou a presença do hipópio com o tamanho da úlcera. Em seu relato, todos os casos de úlcera corneana micótica com diâmetro maior que seis milímetros apresentaram hipópio.

Em relação à etiologia das úlceras, Fusarium sp foi o agente mais encontrado seguido pelo Aspergillus sp, o que é compatível com outros levantamentos realizados em regiões de clima quente ${ }^{(2-3,6,8-11,14,23-25,28)}$. Entretanto, além das variações climáticas, também é importante avaliar o grau de exposição aos fatores associados.

Após serem admitidos no serviço oferecido pelo Hospital, o medicamento mais utilizado foi a natamicina a $5 \%$ devido à sua baixa toxicidade e boa efetividade quanto aos fungos filamentosos, como constatado em outros estudos ${ }^{(2,3,5-6,8-89,13)}$. Contudo, devido à sua penetração corneana insatisfatória, falhas no tratamento com esta droga podem ocorrer.

No presente estudo, o tratamento com natamicina tópica a 


\begin{tabular}{|c|c|c|c|c|c|c|c|c|c|c|}
\hline $\mathbf{N}$ & Idade & Sexo & Trauma & Profissão & Etiologia & $\begin{array}{l}\text { Terapia } \\
\text { prévia }\end{array}$ & $\begin{array}{l}\text { Antifúngico } \\
\text { tópico }\end{array}$ & $\begin{array}{l}\text { Antifúngico } \\
\text { sistêmico }\end{array}$ & CPT & $\begin{array}{c}\text { Recidiva } \\
\text { no enxerto }\end{array}$ \\
\hline 1 & 38 & $\mathrm{M}$ & - & lavrador & Fusarium $s p$ & $\operatorname{sim}$ & natamicina & cetoconazol & $\operatorname{sim}$ & - \\
\hline 2 & 9 & $\mathrm{~F}$ & sim & estudante & Fusarium $s p$ & $\operatorname{sim}$ & natamicina & itraconazol & - & - \\
\hline 3 & 18 & $\mathrm{M}$ & sim & estudante & Fusarium $s p$ & $\operatorname{sim}$ & natamicina & cetoconazol & - & - \\
\hline 4 & 31 & $M$ & sim & lavrador & Fusarium $s p$ & sim & natamicina & - & $\operatorname{sim}$ & - \\
\hline 5 & 20 & M & sim & lavrador & Fusarium $s p$ & sim & natamicina & cetoconazol & - & - \\
\hline 6 & 36 & $\mathrm{M}$ & sim & lavrador & Fusarium sp & sim & $\begin{array}{c}\text { natamicina } \\
\text { anfotericina } \mathrm{B}\end{array}$ & cetoconazol & sim & $\operatorname{sim}$ \\
\hline 7 & 27 & $\mathrm{M}$ & - & pedreiro & Fusarium $s p$ & $\operatorname{sim}$ & natamicina & cetoconazol & $\operatorname{sim}$ & - \\
\hline 8 & 29 & M & sim & lavrador & Fusarium $s p$ & sim & natamicina & - & - & - \\
\hline 9 & 29 & $\mathrm{~F}$ & - & do lar & Fusarium $s p$ & $\operatorname{sim}$ & $\begin{array}{c}\text { natamicina } \\
\text { anfotericina B }\end{array}$ & cetoconazol & $\operatorname{sim}$ & sim \\
\hline 10 & 32 & M & sim & lavrador & Acremonium sp & - & $\begin{array}{c}\text { anfotericina B } \\
\text { intravítrea }\end{array}$ & $\begin{array}{c}\text { itraconazol } \\
\text { anfotericina B EV }\end{array}$ & $\operatorname{sim}$ & - \\
\hline 11 & 47 & M & - & lavrador & Drechsclera $s p$ & $\operatorname{sim}$ & $\begin{array}{c}\text { natamicina } \\
\text { anfotericina B }\end{array}$ & cetoconazol & - & - \\
\hline 12 & 64 & M & - & aposentado & Aspergillus $s p$ & $\operatorname{sim}$ & $\begin{array}{c}\text { miconazol } \\
\text { anfotericina B }\end{array}$ & itraconazol & $\operatorname{sim}$ & sim \\
\hline 13 & 42 & $\mathrm{~F}$ & $\operatorname{sim}$ & do lar & Aspergillus $s p$ & $\operatorname{sim}$ & natamicina & itraconazol & $\operatorname{sim}$ & $\operatorname{sim}$ \\
\hline 14 & 35 & $\mathrm{~F}$ & - & do lar & Aspergillus $s p$ & sim & $\begin{array}{c}\text { anfotericina B } \\
\text { intravítrea }\end{array}$ & cetoconazol & $\operatorname{sim}$ & - \\
\hline 15 & 38 & M & $\operatorname{sim}$ & lavrador & Aspergillus $s p$ & $\operatorname{sim}$ & natamicina & cetoconazol & $\operatorname{sim}$ & $\operatorname{sim}$ \\
\hline 16 & 58 & M & $\operatorname{sim}$ & lavrador & Aspergillus $s p$ & $\operatorname{sim}$ & natamicina & cetoconazol & $\operatorname{sim}$ & $\operatorname{sim}$ \\
\hline 17 & 43 & M & $\operatorname{sim}$ & lavrador & Aspergillus $s p$ & $\operatorname{sim}$ & natamicina & cetoconazol & $\operatorname{sim}$ & - \\
\hline 18 & 67 & M & $\operatorname{sim}$ & lavrador & Fusarium $s p$ & - & natamicina & - & $\operatorname{sim}$ & - \\
\hline 19 & 13 & $\mathrm{~F}$ & - & estudante & Fusarium $s p$ & $\operatorname{sim}$ & natamicina & - & - & - \\
\hline 20 & 38 & M & - & lavrador & Fusarium $s p$ & - & $\begin{array}{c}\text { natamicina } \\
\text { anfotericina } \mathrm{B}\end{array}$ & cetoconazol & $\operatorname{sim}$ & $\operatorname{sim}$ \\
\hline
\end{tabular}

$5 \%$ associada ao antifúngico sistêmico foi eficaz em 13 (76,5\%) pacientes. A necessidade de substituição pelo colírio de anfotericina $\mathrm{B}$ ocorreu em quatro $(23,5 \%)$ pacientes por não apresentarem boa resposta ao tratamento inicial com a natamicina ou por recidiva da infecção micótica após a realização do transplante corneano. $\mathrm{O}$ único paciente inicialmente tratado com miconazol tópico também teve sua prescrição alterada para a anfotericina $\mathrm{B}$ tópica devido à recidiva da infecção micótica no enxerto corneano após o transplante. O tempo de duração do tratamento tópico com a natamicina durou em média 76,5 dias o que se mostrou mais prolongado em relação ao descrito em outras publicações, em função da gravidade dos casos e à lenta melhora das úlceras fúngicas ${ }^{(3,6)}$.

$\mathrm{O}$ uso de medicação anti-fúngica sistêmica em $80 \%$ dos casos pesquisados foi também face à maior gravidade do quadro clínico encontrado nesses olhos ${ }^{(2,6,8)}$. O cetaconazol foi a droga de escolha, pois apresenta boa efetividade e menor custo. A literatura descreve a presença de concentrações altas do princípio ativo desta droga na córnea e na câmara anterior após sua administração oral ${ }^{(2)}$. Itraconazol foi indicado em casos de resposta lenta ao tratamento inicial ou em casos de intolerância ao cetoconazol. A anfotericina B sistêmica foi usada em um paciente que não apresentou melhora com os tratamentos anteriores e apresentava inflação intra- ocular. Esta mesma droga foi aplicada por via intravítrea em dois casos diagnosticados como endoftalmite.

A ceratoplastia penetrante terapêutica foi realizada em 14 (70\%) pacientes, sendo que, em treze $(92,8)$ deveu-se à perfuração das úlceras, o que é um percentual elevado em relação ao encontrado em outras publicações que relatam casos provenientes de regiões com características climáticas semelhantes ${ }^{(6,8,25)}$. Justifica-se tal ocorrência pelas dificuldades de diagnóstico e tratamento e também pelo fato de ser a ceratoplastia penetrante a conduta cirúrgica do serviço quando esta se faz necessária. O transplante corneano é indicado com o objetivo de remover o tecido envolvido e preservar a integridade do globo, ainda assim, os fungos podem permanecer nas bordas do tecido corneano ou na câmara anterior levando a recidivas da infecção micótica ${ }^{(5)}$.

Em três casos $(41,9 \%)$, houve indicação de um segundo transplante corneano terapêutico devido à recidiva da infecção e ausência de resposta ao tratamento clínico e, em um caso $(14,2 \%)$, foi indicado recobrimento conjuntival parcial devido à localização periférica da lesão corneana recidivada com resolução do quadro.

Destes três pacientes, em um caso ainda foi indicado um terceiro transplante corneano que foi seguido de recobrimento conjuntival total também com resolução do quadro infeccioso. 


\section{ABSTRACT}

Purpose: To present 20 cases of fungal keratitis at the São Geraldo Hospital, Belo Horizonte, Minas Gerais, Brazil and to analyze them regarding associated factors, type of identified fungi and treatment. Methods: The authors retrospectively analyzed the charts of 20 patients ( 20 eyes) of culture positive fungal keratitis treated from January 1994 to December 1999 at the São Geraldo Hospital. Results: Fifteen patients (75\%) were male. The average was 35.7 years (range 9 to 67 years). One of the associeted factors analyzed was ocular trauma (60\%). Fusarium sp was the most commonly isolated organism (60\%), followed by Aspergillus sp (30\%). Natamycin was the topical antifungals most frequently used, while systemic treatment commonly used was cetoconazol. Fourteen patients (70\%) had penetrating keratoplasty during the acute stage of infection. Conclusions: We found Fusarium sp the most commonly isolated fungus and a past history of ocular trauma was common. In contrast to other studies, our experience showed a higher number of therapeutic penetrating keratoplasty.

Keywords: Keratitis/drug therapy; Eye infections fungal/drug therapy; Cornea/injuries

\section{REFERÊNCIAS}

1. Leber TH. Keratomycosis aspergillina als ursache von hypopyonkeratites. Graefes Ach Clin Exp Ophthalmol 1879;25:285-301.

2. Foster CS. Fungal keratitis. Infect Dis Clin North Am 1992;6:851-7.

3. Jones DB, Sexton R, Rebell G. Mycotic keratitis in South Florida: a review of thirty-nine cases. Trans Ophthalmol Soc UK 1970;89:781-97.

4. Thygeson P, Okumoto M. Keratomycosis: a preventable disease. Trans Am Acad Ophthalmol Otolaryngol 1974;78:OP433-9.

5. Chin GN, Hyndiuk RA, Kwasny G, Schultz RO. Keratomycosis in Wisconsin. Am J Ophthalmol 1975;79:121-5.

6. Rosa RH, Miller D, Alfonso EC. The changing spectrum of fungal keratitis in South Florida. Ophthalmology 1994;101:1005-13.

7. Kelly LD, Pavan-Langston D, Baker AS. Keratomycosis in a New England referral Center: spectrum of pathogenic organisms and predisposing factors. In: Bialasiewics AA, Schall KP, editors. Infectious diseases of the eye. Stoneham: Butterworth-Heinemann; 1994. p.184-90.
8. Tanure MA, Cohen EJ, Sudesh S, Rapuano CJ, Laibson PR. Spectrum of fungal keratitis at Wills Eye Hospital, Philadelphia, Pennsylvania. Córnea 2000;19:307-12.

9. Vieira LA. Ceratite micótica. In: Belfort Jr R, Kara-José N. Córnea Clínicacirúrgica. São Paulo: Roca; 1997. p.189-203.

10. Liesegang TJ, Forster RK. Spectrum of microbial keratitis in South Florida. Am J Ophthalmol 1980;90:38-47.

11. Andrade AJM, Vieira LA, Höfling Lima AL, Yu MCZ, Gompertz OF, Freitas $\mathrm{D}$ et al. Laboratorial analyses of fungal keratitis in a University Service. Arq Bras Oftalmol 2000;63:59-63.

12. Vieira LA, Belfort Jr R, Fischman OF, Scarpi M. Estudo da flora fúngica da conjuntiva normal, da cana de açúcar e de anemófilos da região canavieira de Santa Rita, Paraíba, Brasil. Arq Bras Oftalmol 1989;52:63-7.

13. Doughman DJ, Leavenworth NM, Campbell RC, Lindstrom RL. Fungal keratitis at the University of Minnesota: 1971-1981. Trans Am Ophthalmol Soc 1982;80:235-47

14. Griffiths MFP, Clayton YM, Dart JKG. Antifungal sensitivity testing of keratitis isolates at Moorfiels Eye Hospital 1975-1990: therapeutic implications. In: Bialasiewics AA, Schall KP, editors. Infectious diseases of the eye. Stoneham: Butterworth-Heinemann; 1994. p.190-4.

15. Wilson LA, Ahearn DG, Jones DB, Sexton RR. Fungi from the normal outer eye. Am J Ophthalmol 1969;67:52-6.

16. Willianson J, Gordon AM, Wood R, Dyer AM, Yahya AO. Fungal flora of the conjunctival sac in health and disease. Br J Ophthalmol 1965;49:505-15.

17. Scarpi MJ. Microbiota fúngica da conjuntiva normal de trabalhadores no corte da cana de açúcar. Rev Bras Oftalmol 1985;44:57-65.

18. Costa ML, Galvão PG, Lage J. Flora micótica da conjuntiva de indivíduos normais. Rev Bras Oftalmol 1965;24:675-82.

19. Hammeke JC, Ellis PP. Mycotic flora of the conjunctiva. Am J Ophthalmol 1955;39:88-92.

20. Ainley R, Smith B. Fungal flora of the conjunctival sac in healthy and diseased eyes. Br J Ophthalmol 1965;49:505-15.

21. Ando N, Takatori K. Fungal flora of the conjunctival sac. Am J Ophthalmol 1982;94:67-74

22. Fedukowicz HB. Clinical and laboratory techniques in external ocular diseases and endopthalmitis. In: Fedukowicz HB. External infections of the eye: bacterial, viral, mycotic with noninfectious and immunologic diseases. New York: Appleton-Century-Crofts; 1985. p.17-81.

23. Srinivasan R, Kanungo R, Goyal JL. Spectrum of oculomycosis in South Índia. Acta Ophthalmol 1991;69:744-9.

24. Khairallah SH, Byrne KA, Tabbara KF. Fungal keratitis in Saudi Arabia. Doc Ophthalmol 1992;79:269-76.

25. Gugnani HC, Talwar RS, Njoku-Obi AN, Kodilinye HC. Mycotic keratitis in Nigeria. Br J Ophthalmol 1976;60:607-13.

26. Mino de Kaspar H, Zouwek G, Paredes ME, et al. Mycotic keratitis in Paraguay. Mycoses 1991;34:251-4.

27. Panda A, Vajpayee RB, Kumar TS. Critical evaluation of therapeutic keratoplasty in cases of keratomycosis. Ann Ophthalmol 1991;23:373-6.

28. Lima ALH, Nishi M, Lottemberg CL, Guiudugli T. Úlceras de córnea em serviço de referência. Arq Bras Oftalmol 1988;51:118-20.

\title{
ABO ELETRÔNICO
}

\author{
Novo site
}

\section{Acesso: http://www.abonet.com.br}

